Trans

continentales
Transcontinentales

Sociétés, idéologies, système mondial

$4 \mid 2007$

Amérique latine

\title{
Virage à gauche et intégration régionale en Amérique latine
}

Shifts to the left and regional integration in Latin America

\section{Olivier Dabène}

\section{(2) OpenEdition}

1 Journals

Édition électronique

URL : http://journals.openedition.org/transcontinentales/595

DOI : 10.4000/transcontinentales.595

ISBN : 978-2-7351-1565-5

ISSN : $1775-397 X$

\section{Éditeur}

Editions de la maison des sciences de l'homme

\section{Édition imprimée}

Date de publication : 1 juin 2007

ISBN : 978-2-200-92396-9

ISSN : 1950-1684

\section{Référence électronique}

Olivier Dabène, "Virage à gauche et intégration régionale en Amérique latine », Transcontinentales [En ligne], 4 | 2007, mis en ligne le 27 avril 2011, consulté le 08 septembre 2020. URL : http://

journals.openedition.org/transcontinentales/595; DOI : https://doi.org/10.4000/transcontinentales. 595

Tous droits réservés 


\title{
Virage à gauche et intégration régionale en Amérique latine
}

\author{
Olivier Dabène
}

Depuis 2002, l'Amérique latine a basculé à gauche. La victoire de Lula au Brésil a servi de détonateur à un mouvement qui s'est tout d'abord étendu aux voisins argentin (Nestor Kirchner en 2003) et uruguayen (Tabaré Vázquez en 2004) et qui aujourd'hui surprend par son ampleur. À l'issue du cycle électoral de 2006, qui a vu le continent procéder à douze élections présidentielles, seuls le Mexique et la Colombie peuvent être considérés comme gouvernés à droite. Partout ailleurs se sont imposés des candidats de gauche modérée (Chili, Brésil, Costa Rica, Pérou) ou radicale (Nicaragua, Bolivie, Équateur, Venezuela). Ces changements politiques se déroulent sur un arrière plan de régionalisme en pleine évolution. Le projet de Zone de libre-échange des Amériques (ZLEA) lancé à l'occasion du sommet des

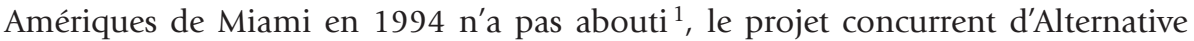
bolivarienne pour les Amériques (ALBA) du président vénézuélien Hugo Chávez ne suscite guère l'unanimité et tarde à prendre forme, tandis que les accords traditionnels se trouvent tous plus ou moins en crise, que ce soit le Système d'intégration centraméricain (SICA), auquel les Centraméricains préfèrent le Traité de libreéchange avec les États-Unis (CAFTA), la Communauté andine (CAN), affaiblie par le retrait du Venezuela, ou le Mercosur, qui peine à se relancer et doit à présent gérer l'adhésion du Venezuela, un nouveau membre quelque peu encombrant. Si la Zone de libre-échange des Amériques (ZLEA) représente clairement un agenda d'intégration d'inspiration néo-libérale, se fixant essentiellement pour objectif d'éliminer les obstacles au commerce et aux investissements dans la région, dans la droite ligne de l'Accord de libre-échange nord-américain (ALENA), l'Alternative bolivarienne pour les Amériques (ALBA) propose de rapprocher les peuples et non les marchés.

1 - Les négociations pour la ZLEA, qui devaient aboutir en 2005, se sont enlisées dès 2001-2002. Toutefois, lors du $4^{e}$ sommet des Amériques de Mar del Plata (novembre 2005), un certain nombre de pays (de fait, tous sauf les membres du Mercosur) ont manifesté le souhait de voir les négociations relancées.

Olivier Dabène : Professeur de science politique à l'Institut d'études politiques de Paris. 
LeS DIVERS ACCORDS D'INTÉGRATION RÉGIONALE EN AMÉRIQUE LATINE eN 2007

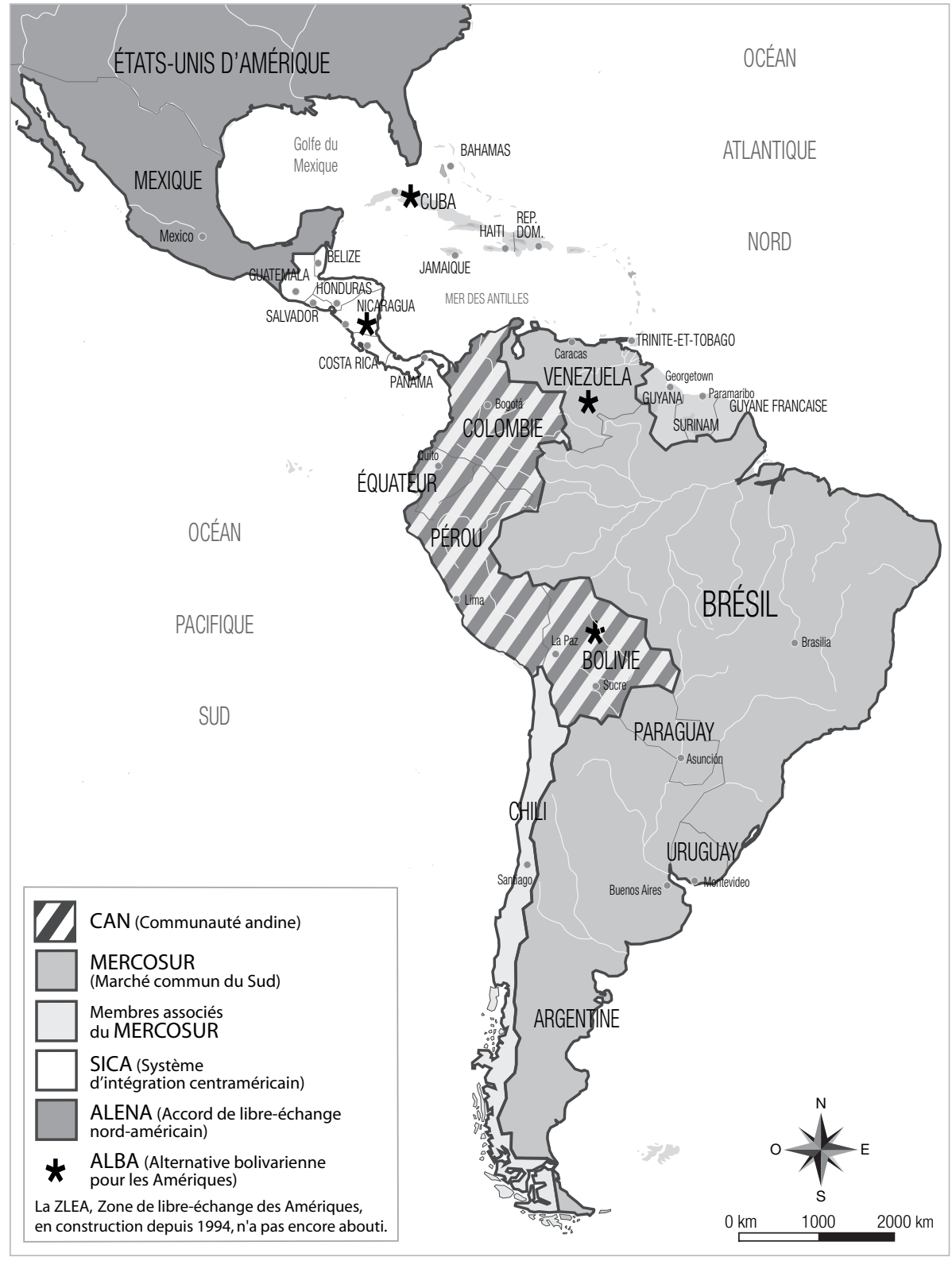

$\mathrm{Au}$ risque de caricaturer quelque peu, on peut avancer que la ZLEA et l'ALBA représentent respectivement des projets d'intégration de droite et de gauche. Le Mercosur, dans ce panorama, tente d'incarner une voie médiane, ne refusant pas les règles du marché, mais tentant de les infléchir à des fins redistributives. Afin de prendre la mesure des changements que le virage à gauche a suscités dans le Mercosur, je commencerai pas quelques rappels historiques, avant de me pencher sur trois réformes engagées depuis 2003. 


\section{Olivier Dabène Virage à gauche et intégration régionale}

\section{Aux origines du projet intégrationniste}

Tandis qu'en Europe la seconde guerre mondiale est propice à une interrogation fondamentale sur les conditions de la paix, l'Amérique latine réfléchit plutôt aux caractéristiques de son développement. Dans les deux cas, l'intégration régionale apparaît comme un instrument de nature à atteindre ces objectifs.

Les ouvrages de David Mitrany, A Working Peace System (1943), et de Raúl Prebisch, The Economic Development of Latin America and its Principal Problem (1949) jettent, des deux côtés de l'Atlantique, les bases d'une certaine conception de l'intégration. Mitrany propose au monde une "alternative fonctionnaliste» qui est à l'origine de la méthode des solidarités concrètes chère à Jean Monnet, tandis que Prebisch, secrétaire général de la Commission économique pour l'Amérique latine et les Caraïbes (CEPALC) à sa création en 1948, propose de stimuler l'industrialisation en la liant au protectionnisme afin de mettre un terme à l'échange inégal et à la détérioration des termes de l'échange. La stratégie d'industrialisation substitutive d'importations (ISI) doit être mise en œuvre à une échelle régionale et faire l'objet d'une planification soucieuse de complémentarité. L'intégration régionale est donc un outil de développement, auquel s'ajoute à la fin des années 1950 une dimension commerciale. En 1955, est créé au sein de la CEPALC un comité du commerce qui à partir des années 1956-1957 réfléchit à la mise en place d'une zone de libreéchange à l'échelle du continent ${ }^{2}$.

En Europe, le projet intégrationniste a été porté à son origine par la démocratie chrétienne (Adenauer, Gasperi, Schuman), tandis qu'en Amérique latine, les idées de Prebisch séduisent les milieux marxistes. Sa théorie est influente. Le courant structuraliste se développe à la CEPALC et se propage grâce aux técnicos qu'elle forme. Son impact politique demeure toutefois modeste, pour deux raisons. D'une part, la bonne tenue des cours mondiaux des matières premières entre 1945 et 1948 puis pendant la guerre de Corée (1950-1954) semble démentir la thèse de Prebisch-Singer, et rend les propositions de la CEPALC peu attractives. Le changement n'intervient qu'après la guerre de Corée (1954), avec une chute des cours. Le projet intégrationniste se développe alors dans la deuxième moitié des années 1950. D'autre part, au plan politique, les nombreux dictateurs du début des années 1950 (Getúlio Vargas au Brésil, Juan Perón en Argentine, Rafael Trujillo en République dominicaine, Manuel A. Odría au Pérou, Marcos Pérez Jiménez au Venezuela) sont opposés à l'idée de la planification (nationale et encore plus internationale) que suppose l'intégration. Là aussi des changements interviennent avec l'arrivée au pouvoir de nouveaux leaders : Arturo Frondizi en Argentine (1958-1962), Juscelino Kubitschek au Brésil (1954-1959), Alberto Lleras Camargo en Colombie (19571962) et Rómulo Betancourt au Venezuela (1958-1964). Ces dirigeants ne sont pas de gauche, et l'on retrouve chez eux à la fois une volonté de rompre le lien de dépendance vis-à-vis des États-Unis (et donc un attrait pour l'ISI de Prebisch), et l'ambition de recevoir une aide comparable au plan Marshall. 
Comme en Europe, ce sont plutôt les courants démocrates-chrétiens (Eduardo Frei au Chili par exemple dans les années 1960), mais aussi sociaux-démocrates (José Figuéres, au Costa Rica), qui portent le projet intégrationniste, à la différence des années 1920-1930, où la réactivation des projets de rapprochements politiques en Amérique latine (et l'anti-impérialisme) était le fait de partis politiques de gauche comme l'Alliance populaire révolutionnaire américaine (APRA) du dirigeant péruvien Haya de la Torre, ou le Parti révolutionnaire institutionnel (PRI) au Mexique, ou encore le Mouvement nationaliste révolutionnaire (MNR) en Bolivie. La création du Parlement latino-américain, le 7 décembre 1964 à Lima, par des délégués de quatorze pays, est le produit de cette convergence politique, dont la gauche est largement absente, parce que réprimée à cette époque de guerre froide et d'Alliance pour le progrès, ce programme d'assistance à l'Amérique latine lancé par le président Kennedy en 1961 pour faire pièce à l'influence de Cuba dans la région.

La première mise en œuvre d'un projet intégrationniste a lieu en Amérique centrale, et elle est empreinte d'ambiguïté. On y retrouve un rôle important joué par les partis politiques démocrates-chrétiens et sociaux-démocrates (José Figuéres, Juan Jose Arévalo), mais aussi par le Parti unioniste (fondé en 1899 au Guatemala). Le climat de solidarité démocratique entre le Salvador et le Guatemala facilite leur rapprochement politique dans les années 1944-1945. Le projet d'intégration politique (ODECA, 1951) est un échec, mais des organismes régionaux comme l'ICAP et l'ICAITI ${ }^{3}$ forment des técnicos et divulguent les thèses de la CEPALC. Toutefois, bien que la CEPALC ait suscité la création d'un Comité de coopération économique de l'isthme centraméricain (CCE) en 1951, le traité tripartite qui va servir de détonateur à l'intégration (Guatemala, Honduras, Salvador) est négocié sans l'appui de la CEPALC, et plutôt sous l'influence des États-Unis qui s'opposent à la planification industrielle. Le traité de 1960 donnant naissance au Marché commun centre-américain (MCCA) s'éloigne donc des thèses de la CEPALC. Le MCCA connaît un certain succès dans les années 1960, mais surtout en termes de dynamisation du commerce réciproque. Le programme d'industrialisation stagne, ou alors profite aux multinationales qui installent des usines de montage à faible valeur ajoutée dans la région. Pendant cette période, l'influence des États-Unis, à travers le bureau pour l'Amérique centrale et Panama (ROCAP), est plus sensible que celle de la CEPALC. Quoi qu'il en soit, le MCCA génère des asymétries qui sont indirectement à l'origine de la guerre de «désintégration régionale ${ }^{4}$ » de 1969 entre le Salvador et le Honduras.

Ailleurs dans le continent, la signature du traité de Rome en mars 1957 en Europe a pour effet d'accélérer la convergence entre deux projets de rapprochements en cours (Venezuela, Colombie et Équateur d'un côté, Argentine, Uruguay, Brésil et Chili de l'autre). En septembre 1957, la Conférence économique de l'Organisation des États américains (OEA) à Buenos Aires recommande la création d'un Marché commun latino-américain. En 1960 est signé le traité de Montevideo créant l'Association

3 - Instituto centroamericano de administración pública (ICAP, 1954) et Instituto centroamericano de investigación y tecnología industrial (ICAITI, 1955)

4 - Alain Rouquié, "Honduras - El Salvador. La guerre de cent heures : un cas de "désintégration" régionale», Revue française de science politique, XXI (6), décembre 1971. 
latino-américaine de libre commerce (ALALC). Traité décevant, puisqu'il ne prévoit aucune obligation réciproque, reflétant les différends politiques entre les pays. Le dialogue diplomatique n'en est pas moins productif, qui se traduit par exemple, lors de la réunion de l'OEA de janvier 1962 à Punta del Este, par l'opposition de six pays à l'expulsion de Cuba (Argentine, Bolivie, Brésil, Chili, Équateur, Mexique), ou encore par le Consensus de Viña de Mar de $1969^{5}$. Le Chili prend l'initiative de recommander une accélération de l'intégration en 1963, année où est créée la Commission spéciale de coordination latino-américaine (CECLA). Après une réunion Alessandri ${ }^{6}$ / Goulart $^{7}$ en avril 1963, Frei réunit un "groupe des 4 " (l'Argentin Raúl Prebisch, le Chilien Felipe Herrera, le Vénézuélien Jose Antonio Mayorbe, le Colombien Carlos Sanz de Santamaria) qui au printemps 1965 propose de créer un marché commun en 1975. Les recommandations du groupe ne sont pas suivies. Mais un sommet des Amériques est convoqué à Punta del Este (12-14 avril 1967). La Déclaration des présidents d'Amérique appelle à la création progressive d'un marché commun. Pour la première fois, les États-Unis s'associent à ce projet. Mais ce sommet n'a pas de suite. L'idée d'intégration a cependant progressé et, dès 1966, les pays andins se réunissent; ils vont mettre en route le Groupe andin en 1969.

Sans entrer dans plus de détails, cette brève évocation historique montre bien que le projet intégrationniste cépalien, que l'on peut légitimement classer à gauche, a été soit ignoré dans les années 1950, soit dévoyé dans les années 1960 où il connut une dérive libre-échangiste et un abandon de l'ambition développementaliste.

Comme nous incite à le penser une perspective en termes de «dépendance du sentier", la conjoncture historique du démarrage de l'intégration compte, au même titre que la séquence historique qui suit et qui crée de l'irréversibilité et des effets de rétroaction positive ${ }^{8}$. En l'occurrence, deux décalages mettent l'intégration sur des rails particuliers : d'un côté, le décalage initial entre la théorie (échange inégal) et l'expérience (prospérité), de l'autre le décalage entre le climat politique porteur des années 1944-1945 et la création de la CEPALC (1948) dans un climat de guerre froide rendant suspecte toute évocation de la nécessité d'une planification du développement industriel. Une fois lancée sur des bases essentiellement commerciales, l'intégration est alimentée par deux mécanismes qui conjuguent leurs effets pour accentuer sa dépolitisation : les técnicos de la CEPALC, travaillant dans les ministères de l'Économie, deviennent des micro-entrepreneurs de l'intégration dépourvus d'une vision globale, et les bons résultats «statistiques» enregistrés (surtout en Amérique centrale) confortent la démarche d'ensemble. Il y a bien là

5 - Document élaboré lors d'une réunion des ministres des Affaires étrangères d'Amérique latine à Santiago du Chili le 17 mai 1969, mettant l'accent sur les problèmes du continent et la nécessaire redéfinition des relations avec les États-Unis.

6 - Jorge Alessandri, président du Chili du 3 novembre 1958 au 3 novembre 1964.

7 - João Marques Goulart, président du Brésil du 7 septembre 1961 au $1^{\mathrm{er}}$ avril 1964, renversé par un putsch militaire.

8 - La notion de «dependance du sentier» (path dependence) permet d'expliciter les mécanismes qui contraignent des choix institutionnels. Une fois un pays engagé dans une trajectoire de développement, toute inflexion a un coût élevé. Voir Paul Pierson, Politics in Time. History, Institutions and Social Analysis, Princeton, Princeton University Press, 2004. 
un exemple de boucle rétroactive positive renforçant une tendance initiale apparue dans une conjoncture très précise.

\section{Le Mercosur, un projet cépalien avorté?}

Le rapprochement entre l'Argentine et le Brésil au milieu des années 1980 n'est pas le fait de gouvernements de gauche. Les militaires avaient pris les devants en développant des projets communs. Mais le passage à la démocratie de l'Argentine (1983) et du Brésil (1985) génère de l'incertitude politique. Et cette incertitude constitue une autre conjoncture critique favorable au démarrage d'un processus d'intégration conçu comme une politique de solidarité démocratique. Le projet, tel qu'il est défini dans le Programme d'intégration et de coopération économique (PICE) prévu dans l'Acte pour l'intégration argentino-brésilienne de 1986, dans les 24 protocoles signés entre juillet 1986 et août 1989, et le Traité d'intégration, coopération et développement (Buenos Aires, 29 novembre 1988), prévoient une ouverture progressive par produit, la promotion d'une intégration intra-industrielle, la coordination et l'harmonisation des politiques macroéconomiques et l'élimination des asymétries. L'ensemble dénote une certaine influence cépalienne.

Mais à nouveau, la séquence historique possède des caractéristiques qui engagent l'intégration dans un "sentier» autre que celui prévu initialement, pour trois séries de raisons. L'objectif de la coordination de politiques macroéconomiques se heurte à l'instabilité qui caractérise la période de l'échec des plans Austral (Argentine) et Cruzado (Brésil). Conséquence de cette instabilité, de nouveaux dirigeants sont par ailleurs élus en 1989-1990 (Carlos Menem en Argentine, Fernando Collor de Mello au Brésil, Luis Alberto Lacalle Herrera en Uruguay) qui opèrent un tournant néolibéral dans la région. Enfin, le 27 juin 1990, le président des États-Unis Georges Bush lance son Initiative pour les Amériques, qui comprend trois volets, un fonds multilatéral à la Banque interaméricaine de développement (BID) pour stimuler l'investissement privé, un dispositif de renégociation de la dette lié à des réformes économiques, et des accords ayant pour objectif le libre-échange au plan continental. Le 6 juillet 1990, l'Argentine et le Brésil signent l'acte de Buenos Aires, qui sanctionne un net changement de méthodologie. Le développement de projets intégrés et la coordination de politiques macroéconomiques sont abandonnés, et l'accent est placé sur le libre-échange. Le traité d'Asunción du 26 mars 1991 donnant naissance au Mercosur est un traité dépolitisé, au double sens d'absence de projet politique et d'institutions. Dix ans après, les pays membres du Mercosur n'ont eu à célébrer que le sauvetage de l'intégration après la dévaluation brésilienne et la crise argentine. De façon intéressante, apparaît alors une volonté de relancer l'intégration qui trouve son origine dans le dialogue entre le président argentin Eduardo Duhalde et son homologue brésilien Luiz Inácio Lula da Silva en novembre 2002, et un diagnostic pointant l'imperfection de l'union douanière, l'inachèvement de la mise en œuvre des normes communautaires et les déficiences institutionnelles. Un retour à l'esprit du Programme d'intégration et de coopération économique est notable. Avec Nestor Kirchner qui succède à Duhalde en 2003, le président uruguayen Tabaré Vázquez et Lula, l'intention initiale est claire : le Mercosur doit se détourner d'une intégration libre-échangiste et évoluer vers une intégration plus positive. En témoignent les 
«Objectifs 2004-2006» adoptés en juin 2003 à Asunción, à l'occasion du premier sommet du Mercosur avec le couple Lula/Kirchner. D'autres documents vont dans le même sens, comme le «Programme pour la consolidation de l'union douanière et pour le lancement du marché commun» ou des déclarations conjointes de Lula/ Kirchner ("Consensus de Buenos Aires», octobre 2003; et "Acte de Copacabana», février 2004). Face aux défis sociaux, l'intégration doit franchir un seuil qualitatif. Différentes réformes sont alors engagées, mais dans un climat pesant dû à d'acerbes différends commerciaux entre l'Argentine et le Brésil qu'une simple clause de sauvegarde aurait pu permettre d'éviter. Le sommet d'Ouro Preto de décembre 2004, qui aurait dû être l'occasion d'une réforme d'envergure à l'occasion du dixième anniversaire du protocole d'Ouro Preto (POP) de 1994, n'a été le théâtre que d'acrimonies.

Trois réformes méritent toutefois un examen attentif: le Secrétariat d'assistance technique, le Parlement et le Fonds de convergence structurelle. Je dégagerai ensuite des enseignements de leur relatif échec.

Le secrétariat du Mercosur, modeste organe administratif, est transformé en 2003 en Secrétariat technique doté d'un secteur d'assistance technique. Sa tâche consiste alors à «contribuer à la formation d'un espace de réflexion commun sur le développement et la consolidation du processus d'intégration ". L'organisation en 2003 d'un concours de recrutement de quatre assistants techniques, deux économistes et deux juristes a bouleversé bien des habitudes. Sélectionnés parmi plus de quatre cents candidats, les quatre nouveaux assistants disposent d'une légitimité que leur confère leur très haute compétence, et qui les pose en position de surplomb par rapport aux diplomates, parfois de piètre niveau, qui ont en main les différentes négociations depuis une quinzaine d'années. Avec ce secteur d'assistance technique, un embryon de défense de l'intérêt commun apparaît pour la première fois. La première année de fonctionnement du Secrétariat d'assistance technique (SAT) a immanquablement été marquée par de nombreux accrochages, notamment avec les secteurs les plus conservateurs de la diplomatie, très attachés au caractère strictement intergouvernemental du Mercosur. Les «ennemis» du SAT sont par exemple parvenus à subordonner le soutien technique apporté aux différents organes consultatifs du Mercosur à l'accord préalable unanime des organes permanents, ce qui risque de contraindre le SAT à exercer ses fonctions de manière plus informelle. Mais les assistants techniques sont parvenus à faire prévaloir leurs points de vue intégrationnistes et à donner à voir la perspective régionale de l'intégration, ce qui leur a permis d'être entendus par les hautes sphères du pouvoir. Ainsi, dans le respect de la décision CMC 30/02 du Conseil du marché commun (CMC), le SAT a élaboré en 2004 son premier rapport semestriel, et a donné à cet exercice le ton critique et prospectif

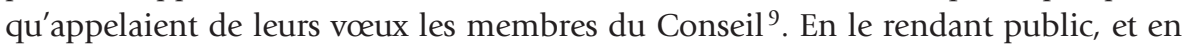
inspirant l'organisation d'un séminaire de réflexion préparatoire au sommet d'Ouro Preto, le SAT est allé plus loin encore, et s'est affirmé comme nouvel entrepreneur politique du Mercosur. Car l'enjeu résidait bien dans la préparation du sommet du

9 - Voir SM/SAT, Un foco para el proceso de integración regional, Primer informe de la Secretaría del Mercosur, Montevideo, juillet 2004. 
Mercosur d'Ouro Preto, du 17 décembre 2004. Après s'être abstenu de célébrer le dixième anniversaire de la signature du traité d'Asunción en 2001, un "deuxième» dixième anniversaire serait célébré par les présidents Lula, Kirchner et Vázquez, tous très attachés aux progrès de l'intégration. Toutefois, l'affrontement entre les experts du SAT et les diplomates tourne rapidement à l'avantage des seconds. Les rapports semestriels et pratiquement tous les avis du SAT tombent sous le sceau de la confidentialité et, finalement, le SAT est remanié, par la décision 07/07, pour lui conférer un profil nettement plus bas. Avec cette révision à la baisse des prérogatives du SAT, le Mercosur se prive d'un «moteur» important. Face à l'implacable utilitarisme de la logique intergouvernementale, le SAT avait commencé à incarner l'intérêt général. Sans doute les gouvernements de gauche, très attachés à la rhétorique intégrationniste, n'ont-ils pas pris la mesure des enjeux. Les diplomates, eux, ont très tôt engagé une guerre d'usure qui, dans le quotidien des micro-rapports de force, a fini par avoir raison du projet. Les quatre experts ont finalement tenté $\mathrm{d}$ 'introduire un type de comportement en rupture avec les règles du jeu de l'intégration, consolidées depuis bien plus longtemps que l'existence du Mercosur. De fait, depuis le rejet dans les années 1960 du sentier cépalien d'intégration qui prévoyait de faire prévaloir un projet régional d'industrialisation, la négociation intergouvernementale s'est imposée comme méthode, et toute possible dérive vers une logique supranationale est étouffée dans l'œuf. L'ALALC, Association latino-américaine de libre commerce (1960), puis l'ALADI, Association latino-américaine d'intégration (1980), qui auraient pu incarner cet intérêt général, ont été reléguées à un rôle de chambres d'enregistrement des accords signés. Cette phobie de la dimension supranationale de l'intégration se donne à voir sur un plan institutionnel, mais aussi et surtout dans les comportements des acteurs, qui ne parviennent pas à se détacher de la représentation d'intérêts nationaux. Les assistants techniques du Mercosur ont pu le faire pendant trois ans, grâce à leur mode de recrutement. Mais paradoxalement, ce mode de recrutement par concours les a aussi privés du type de soutien politique que garantit un mode de nomination plus clientéliste.

Autre réforme importante et emblématique du Mercosur, la création d'un Parlement était envisagée dès les débuts du processus, à la fois pour le doter de légitimité démocratique et d'efficacité. L'implication des parlementaires, et donc des classes politiques nationales, était censée pouvoir faciliter l'introduction des normes communautaires dans les législations nationales. Le traité d'Asunción de 1991 prévoyait, dans son article 24, la création d'une Commission parlementaire du Mercosur, afin de «faciliter l'avancée vers la formation d'un marché commun». En décembre 1991, la Commission parlementaire conjointe (CPC) est installée et son règlement intérieur approuvé. La CPC est composée de soixante-quatre membres, regroupés en quatre sections nationales de seize parlementaires chacune. L'article 3 de son règlement intérieur mentionne que la Commission aura un «caractère consultatif, délibératif et de formulation de déclarations, dispositions et recommandations». Par ailleurs, au titre de ses attributions, figure le «développement d'activités nécessaires pour faciliter la future installation du Parlement du Mercosur». Le protocole d'Ouro Preto (POP) inclut la CPC dans la structure organique du Mercosur, mais dans une position relativement subalterne, puisqu'elle ne fait pas partie des organes dotés de capacités de décision. Le POP dispose que la CPC émet des recommandations au 
Conseil du marché commun, par l'intermédiaire du Groupe du marché commun. De surcroît, aucun organe du Mercosur ne prévoit de "consulter» la CPC, en sorte que le "caractère consultatif» auto-attribué par la CPC n'a jamais pu se développer. Les changements politiques évoqués plus haut créent une structure d'opportunités nouvelles, et le 15 décembre 2003, le Conseil du marché commun (CMC) approuve son programme de travail pour 2004-2006, dans lequel il évoque la création du Parlement pour fin 2006. Auparavant, avait été signé le 6 octobre 2003 un accord inter-institutionnel Commission parlementaire conjointe/Conseil du marché commun, appelé "amendement Alonso ", qui prévoyait de renforcer la participation des parlements nationaux à la «conformation de l'ordre juridique du Mercosur». Dans ce cadre, la CPC doit servir de «laboratoire réel et effectif pour profiler le rôle du futur Parlement régional». De son côté, le CMC s'engageait, dans cet accord, à «consulter la CPC pour tous les sujets qui requièrent l'approbation législative pour son incorporation dans les ordres juridiques des États membres» (article 1). En échange, la CPC s'engage à «impulser, à travers ses sections nationales, un travail responsable d'internalisation de la norme Mercosur» (article 2). Cet accord interinstitutionnel n'a pas produit les effets escomptés. À ce stade, il est intéressant de noter que la $\mathrm{CPC}$, en dépit de sa capacité à rédiger son propre règlement intérieur, n'a pas su évoluer en 1992 et 1999 vers une affirmation de son rôle politique, comme l'avait fait l'Assemblée européenne avant de se convertir en Parlement en 1979. Pas plus que l'accord inter-institutionnel de 2003 n'a pu contribuer à cette affirmation. En juillet 2004, la Commission parlementaire conjointe présente un projet de parlement au Conseil du marché commun, qui doit être inclus dans un "paquet» de réformes devant rafraîchir le protocole d'Ouro Preto et relancer le Mercosur. Le sommet d'Ouro Preto de décembre 2004 est pourtant gâché par les différends commerciaux entre l'Argentine et le Brésil et le POP n'est pas réformé. Il faut attendre un an de plus (9 décembre 2005) pour que soit approuvé le protocole constitutif du Parlement du Mercosur. Au total, le Parlement est une institution modeste, composé de dix-huit représentants par pays (qui seront élus à partir de 2010), dont la fonction principale consiste à prononcer un avis sur des projets de normes. L'organe de prise de décision peut ne pas suivre l'avis du Parlement, mais s'il le fait la procédure est accélérée (fast track). Le Parlement peut aussi demander des rapports d'activité aux différents organes. Là encore, il apparaît clairement que les États membres n'ont pas osé franchir le seuil de la supranationalité et de l'émergence de la représentation d'un intérêt général. Les députés sont répartis en sections nationales, et non en groupes parlementaires, et le Parlement est dépourvu de fonction de codécision. L'exemple européen est là pour montrer que les pratiques peuvent anticiper sur les règles, et qu'une Assemblée peut ainsi monter en puissance. Mais l'absence, relative, de tradition parlementaire dans les pays du Mercosur, et le précédent médiocre de la Commission parlementaire conjointe, amènent à douter qu'un tel scénario puisse s'y reproduire.

Dernière illustration de la volonté de réformer le Mercosur, la prise en compte des asymétries est sans doute un enjeu crucial. L'abandon de la voie volontariste de l'intégration positive dans sa version cépalienne avait conduit à se priver d'instruments pouvant garantir la répartition équitable des bénéfices de l'accélération des échanges. Et, de fait, la question des asymétries a été à l'origine, en 1969, à la fois 
d'une guerre de désintégration en Amérique centrale et de la création du Groupe andin. Dans le Mercosur, la croyance toute libérale dans les bienfaits de la libéralisation des échanges a conduit à une évocation timide du thème. L'article 6 du traité d'Asunción «reconnaît différents rythmes ponctuels pour le Paraguay et l'Uruguay, qui sont pris en compte dans le programme de libération commerciale», et dans ses attendus, le protocole d'Ouro Preto mentionne "la nécessité d'une considération spéciale pour les pays et régions de moindre développement du Mercosur». Aucun mécanisme spécifique n'a pourtant été prévu pour compenser des inégalités de développement qui, au regard d'une certaine littérature théorique, constituent un sérieux handicap pour le succès de l'intégration régionale dans le Mercosur ${ }^{10}$. Et en dix ans, comme l'indique ce tableau, ces inégalités se sont plutôt creusées. Ainsi le PIB du Brésil est-il passé de 49 à 53 fois celui du Paraguay.

Asymétries dans le Mercosur : comparaisons 1992/2004*

\begin{tabular}{l|l|l|l|l} 
& Argentine & Brésil & Paraguay & Uruguay \\
\hline PIB en 1992 & 21,7 & 49 & 1 & 1,3 \\
\hline PIB en 2004 & 17,6 & 53 & 1 & 1,1 \\
\hline Exportations en 1992 & 20,6 & 61,9 & 1 & 2,9 \\
\hline Exportations en 2004 & 21,1 & 59,3 & 1 & 1,8
\end{tabular}

* Dans ce tableau, le Paraguay a été pris comme référence (facteur 1)

Ce thème des asymétries prend une connotation toute particulière dans le Mercosur, dans la mesure où le pays le plus riche (le Brésil) comprend les régions les plus pauvres de la zone (Nord et Nord-Est). Tout mécanisme de solidarité régionale calqué sur le modèle européen risque de se heurter à une opposition de bon sens du Brésil : pourquoi alimenter majoritairement un fond régional pour en recevoir les bénéfices? Le Brésil peut légitimement objecter que les asymétries de développement sur son territoire sont bien supérieures à ce qu'elles peuvent être au sein du Mercosur, et que l'homogénéité du développement est donc une préoccupation de politique intérieure. La nouvelle fenêtre d'opportunité politique, évoquée à plusieurs reprises déjà dans cet article, semble dans ce domaine aussi avoir aplani les difficultés. Il n'y a de toute façon guère de relance possible sans contenter les "petits » États membres. En 2004, le Conseil du marché commun (décision n ${ }^{\circ}$ 19/04) crée un Groupe de haut niveau sur la convergence structurelle et le financement du processus d'intégration (GANCEFI), dont la mission consiste à réfléchir à deux problèmes apparemment distincts : la lutte contre les inégalités de développement d'une part, et le financement de l'intégration de l'autre, le second risquant en réalité de limiter les effets recherchés par le premier. Il y a d'un côté un souci concernant le financement du développement des activités du Mercosur, notamment de son secrétariat, et de l'autre un problème de fond : lutter contre les déséquilibres, en prenant

10 - Ernst Haas et Philippe Schmitter, «Economic and Differential Patterns of Political Integration : Projections about Unity in Latin America», International Organization, XVIII (4), 1964. 
en compte l'exemple européen des Fonds structurels, et atteindre un haut degré de cohésion sociale et économique. Sur la base des travaux du groupe de haut niveau, le Conseil du marché commun décide ( $\left.\mathrm{CMC} \mathrm{n}{ }^{\circ} 45 / 04\right)$ de créer un Fonds pour la convergence structurelle du Mercosur (FOCEM), qui doit financer ou cofinancer des projets qui poursuivent un des quatre objectifs suivants :

- programme 1 : réduire les déséquilibres et asymétries régionales et nationales;

- programme 2 : améliorer la compétitivité régionale et nationale;

- programme 3 : promouvoir les conditions du développement social;

- programme 4 : financer les organes de la structure institutionnelle du Mercosur.

Début 2005, l'Uruguay (avec une nouvelle équipe, à la suite de l'élection de Tabaré Vázquez) propose que le secrétariat du Mercosur soit chargé de gérer le FOCEM, ce que l'Argentine conteste, argumentant que cela donnerait au secrétariat un caractère politique et le bureaucratiserait. Les débats aboutissent à deux décisions. La première est prise à l'occasion du sommet d'Asunción du 19 juin 2005. Intitulée «Intégration et fonctionnement du Fonds pour la convergence structurelle et le renforcement de la structure institutionnelle du Mercosur", elle a pour effet de mettre en marche le FOCEM en lui attribuant un apport annuel de 100 millions de dollars. Les apports et bénéfices respectifs des pays sont proportionnels au PIB régional :

\section{RÉPARTITION DES APPORTS ET BÉNÉFICES DU FOCEM}

\begin{tabular}{l|l|l|l|l|} 
& Brésil & Argentine & Uruguay & Paraguay \\
\hline Apports & $70 \%$ & $27 \%$ & $2 \%$ & $1 \%$ \\
\hline Bénéfices & $10 \%$ & $10 \%$ & $32 \%$ & $48 \%$ \\
\hline
\end{tabular}

Il est ajouté que les pays doivent participer aux projets les concernant à hauteur de $15 \%$. Concernant les programmes, il est aussi spécifié (articles 12 et 13) que les financements iront pendant les quatre premières années prioritairement (sans pourcentage) au programme 1 (et tout spécialement à l'infrastructure), et que le programme 4 bénéficiera d'un maximum de $0,5 \%$ du Fonds. Il est enfin précisé que le FOCEM commencera à travailler avec la «méthodologie de projets pilotes à fort impact sur les citoyens du Mercosur». La deuxième décision, prise lors du sommet de Montevideo du 8 décembre 2005, concerne l'approbation du règlement du FOCEM, élaboré par le groupe de haut niveau. Ce règlement mentionne, et c'est un point important, que le FOCEM sera administré par le secrétariat du Mercosur, ce qui est une victoire pour l'Uruguay et pour le renforcement de cette institution. Le Secrétariat sera à cet effet doté d'une unité technique FOCEM (UTF), qui comprendra quatre personnes. Mais dans le même temps, le dispositif prévoit des contreparties dans chaque État membre, sous la forme d'Unités techniques nationales FOCEM (UTNF). Ces unités seront chargées de préparer les projets et de gérer les fonds lorsque les projets sont approuvés. Au total, le MERCOSUR a réalisé un important progrès avec la création du FOCEM. On voit pourtant que ce Fonds englobe sous la préoccupation de solidarité, trois objectifs distincts : réduire les asymétries de développement entre États membres et entre les régions, augmenter la compétitivité de l'union douanière et doter la structure institutionnelle d'un financement stable. Le premier objectif 
répond bien à une ambition de solidarité. Le second un peu moins et le troisième pas du tout. Sauf à considérer que la compétitivité et l'efficacité institutionnelle permettront au Mercosur d'améliorer sa «rentabilité sociale» (l'expression est employée dans l'article32.e du règlement du FOCEM).

\section{Une gauche mercosurienne souverainiste?}

Les trois réformes engagées se heurtent à l'obstacle de la supranationalité, que la gauche mercosurienne ne parvient pas à se résoudre à franchir. Engageant une sorte de pas de tango, les gouvernements ont fait succéder à chaque pas en avant deux pas en arrière, ce qui interdit aux réformes de donner leur pleine mesure. La défense de la souveraineté et l'arrimage à la logique intergouvernementale ont eu raison de l'impulsion initiale donnée par le Secrétariat technique, ont coupé les ailes du Parlement, ont infléchi la logique des fonds structurels, et ont modelé une curieuse méthode d'attribution des fonds structurels (pré-affectation des bénéfices par pays, création d'Unités techniques nationales). La souveraineté ne saurait être cédée au Secrétariat, ni même partagée au sein d'un Parlement. Certains secteurs de gauche ayant pris au sérieux le discours sur la démocratisation du Mercosur et la solidarité en son sein ne peuvent qu'être déçus par ces demi-mesures. Deux remarques restent toutefois à faire qui doivent atténuer ces regrets. La résistance aux cessions ou partages de souveraineté peut tout d'abord très bien se justifier au nom de projets «de gauche». Rien n'indique en effet que l'intérêt général du Mercosur qui serait défendu par un dispositif supranational contribuerait à atteindre les objectifs sociaux que se sont fixés les gouvernements de gauche du Mercosur. Le Brésil de Lula peut après tout très légitimement privilégier son traitement national des inégalités et de la pauvreté, et chercher à tout prix à préserver pour cela ses marges de manœuvre au plan international. Il le fait dans le Mercosur comme il le fait dans les arènes de négociations internationales, du type OMC par exemple. Mais la défense de la souveraineté est aussi le produit d'une "diplomatie à double tranchant (double edge diplomacy) ${ }^{11}$ " bien enracinée, en vertu de laquelle des dispositifs de construction des préférences nationales se sont consolidés, faisant intervenir de puissants groupes de pression patronaux. La Fédération des industriels de l'État de São Paulo (FIESP), pour ne prendre qu'un exemple, sait faire entendre sa voix à Brasília, et n'est sans doute pas prête à accepter que son lobbying reste sans effet. Il y a là un mécanisme lourd, sur lequel un gouvernement de gauche n'a guère prise. La gauche mercosurienne n'est en ce sens pas nécessairement souverainiste. Elle se coule tout simplement, avec plus ou mois de réticence, dans le moule des dispositifs de fabrique de la politique étrangère qui jouent en faveur de la défense de la souveraineté. Enfin, concernant le contenu des réformes, il convient de remarquer qu'en dépit des limitations évoquées plus haut, la gauche mercosurienne a mis en place une politique de solidarité. Le FOCEM n'est pas le premier fonds commun que le Mercosur redistribue ${ }^{12}$ et il ne représente que 0,03\% du PIB de la région, mais il instaure un dispositif permanent. Le retour en vogue des politiques redistributives à

11 - Peter Evans (dir.), Double-Edge Diplomacy, Berkeley, University of California Press, 1993.

12 - Au sommet d'Ouro Preto de décembre 2004, un Fonds de financement de l'éducation (FEM) a été approuvé, d'un modeste montant initial de 360000 dollars. 
usage interne, dans le sillage du programme Bourse famille de Lula, trouve un équivalent au plan régional, pour la première fois en Amérique latine.

Si la redistribution constitue une partie importante de l'identité de la gauche, alors le Mercosur a franchi une étape essentielle vers une intégration positive, tournée vers les outcomes, la démo-distribution de Sartori ${ }^{13}$. Naturellement, ce biais redistributif place aussi le Mercosur dans une posture de gauche réformatrice, largement critiquée par la gauche radicale incarnée par Hugo Chávez, mais aussi par l'administration conservatrice aux États-Unis.

\section{Le Mercosur au défi de la gauche radicale et de la droite}

Hugo Chávez n'a en effet de cesse de critiquer un Mercosur à son goût beaucoup trop néo-libéral. Au-delà du Mercosur, Chávez fustige l'intégration qui repose sur le libreéchange, comme l'incarne le projet de Zone de libre-échange des Amériques (ZLEA), et propose une Alternative bolivarienne (ALBA). Il est à présent en mesure de peser sur l'évolution du Mercosur de l'intérieur, depuis son adhésion précipitée de décembre 2005. Il n'est pas question ici de discuter en détail le projet d'Alternative bolivarienne pour les Amériques (ALBA). Je me limiterai à soulever une question. Peut-on avancer que l'ALBA représente une intégration plus à gauche que le Mercosur (ou que le reste des processus, dans les Andes ou en Amérique centrale)? Au-delà de la rhétorique sur la substitution d'une intégration se fondant sur la solidarité entre les peuples à une intégration se basant sur le libre jeu du marché, force est de constater que la première, et plus tangible, matérialisation de l'ALBA se situe dans le domaine énergétique où Chávez fait preuve d'ambition géopolitique et d'une indéniable générosité. Sa proposition de créer une Banque du Sud relève d'une volonté de soustraire la région à l'influence des bailleurs de fonds, mais n'indique rien sur l'usage qui sera fait des financements.

Si l'on juge qu'une intégration menée par la gauche doit mobiliser des ressources afin de défendre un modèle social, alors l'ALBA doit encore faire ses preuves. Et les projets envisagés pour rapprocher les peuples ne peuvent entrer en concurrence avec la méthodologie du Mercosur. Le défi que représente le modèle d'intégration défendu par les États-Unis est plus sérieux. Rappelons tout d'abord que le Mercosur est le produit des années 1990 dominées par le consensus de Washington. Pour autant, il s'écarte sensiblement du modèle d'intégration nord-américain représenté par l'ALENA ${ }^{14}$. Le Mercosur représente une méthode d'intégration médiane, éloignée du projet cépalien, mais qui ne met pas en péril l'interventionnisme étatique, surtout présent au Brésil. Lorsque le bloc régional s'est trouvé mis au défi de s'adapter aux "nouveaux» thèmes des négociations commerciales (investissements, marchés publics, etc.) dans le cadre du projet de Zone de libre-échange des Amériques (ZLEA), des résistances se sont faites sentir. L'adjudication des marchés publics est,

13 - Giovanni Sartori, The Theory of Democracy Revisited, Chatham, Chatham House Publishers, 1987.

14 - L'ALENA (Accord de libre-échange nord-américain) possède deux caractéristiques importantes : il s'agit d'un accord qui se limite à libéraliser les échanges et à protéger les investissements, et qui inclut des thèmes nouveaux comme les marchés publics et la propriété intellectuelle, voire très nouveaux comme le droit du travail et l'environnement. 
par exemple, un thème intéressant parce qu'il véhicule une conception du rôle de l'État, souvent gravée dans le marbre des Constitutions. Le Brésil a clairement utilisé la passation des marchés publics comme outil de politique industrielle, et avec une certaine réussite. Le refus de démanteler un appareil d'État interventionniste, déjà perceptible sous Cardoso ${ }^{15}$, a été confirmé et accentué par Lula à partir de 2002. La gauche en Argentine, et surtout en Uruguay, n'a pas les mêmes préoccupations. Une partie de la gauche uruguayenne, profondément déçue par le Mercosur, n’hésite même pas à envisager la signature d'un accord de libre-échange avec les États-Unis. Le Mercosur apparaît sur ces questions comme un modèle d'intégration hybride, ce qui ne doit pas surprendre car il est le produit de la synthèse approximative de différentes conceptions du rôle de l'État, l'Argentine étant à cet égard plus libérale que le Brésil.

\section{Conclusion}

Il apparaît en définitive que le Mercosur gouverné par la gauche s'est inscrit dans une certaine continuité par rapport aux orientations données dans les années 1990 par des chefs d'État plus à droite. La gauche est tout autant réticente que la droite face à toute évolution susceptible de faire franchir à l'intégration le seuil fatidique de la supranationalité, mais elle n'en porte pas l'entière responsabilité, car le processus d'intégration génère déjà une inertie difficile à infléchir. Pour autant, la gauche a su doter le Mercosur de capacités redistributives (FEM et FOCEM), ce qui correspond à une évolution conforme à ses projets.

Au-delà du Mercosur, la même ambiguïté est perceptible, qu'il est pareillement difficile d'attribuer à une absence d'enthousiasme ou à des pesanteurs institutionnelles encore plus marquées dans la Communauté andine ou dans le Système d'intégration centraméricain, deux processus d'intégration beaucoup plus anciens que le Mercosur. Dans les Andes, après le retrait de Chávez, la Communauté andine traverse une nouvelle crise. Les deux nouveaux présidents de gauche, le bolivien Evo Morales et l'équatorien Rafael Correa, ont manifesté leur attachement à l'intégration, mais semblent hésiter entre la relance de la Communauté et son abandon pur et simple, pour rejoindre le Mercosur et/ou renforcer l'ALBA. En Amérique centrale, le «revenant» Daniel Ortega, de nouveau président du Nicaragua dix-sept ans après avoir quitté le pouvoir, a confirmé à la fois son attachement à l'intégration régionale, et son attrait pour l'ALBA, mais il n'a pas annoncé qu'il dénoncerait le Traité de libre-échange signé avec les États-Unis par son prédécesseur.

L'ambiguïté règne donc et, en dehors de l'ALBA, il n'y a pas une politique d'intégration de gauche en Amérique latine. Mais même l'ALBA paraît suspecte, évaluée à l'aune de ses projets centrés sur l'énergie. Si le projet cépalien structuraliste sert de référence en matière d'intégration de gauche, alors l'ALBA, sous réserve d'inventaire plus précis, semble pour l'heure plus relever d'une politique d'assistance que d'un projet de développement.

15 - Fernando Henrique Cardoso a été président du Brésil entre 1994 et 2002. 